\title{
Aortic aneurysm and dissection are not associated with an increased risk for giant cell arteritis/ polymyalgia rheumatica
}

\author{
Michael Ehrenfeld, Rafael Bitzur, Jacob Schneiderman, Aram Smolinsky, Yechezkel Sidi, \\ Hanan Gur
}

\begin{abstract}
It has recently been claimed that giant cell arteritis (GCA) is associated with a markedly increased risk of aortic aneurysm formation or rupture. In the present study, the opposite approach was taken, by looking for the incidence of GCA and polymyalgia rheumatica (PMR) in patients with aortic aneurysm, aortic dissection, or both (AA/D). The records of 315 consecutive patients admitted with the diagnosis of AA/D were reviewed. In addition, follow up information was obtained in 82 patients by examination in the outpatient clinic. After careful examination and assessment of clinical and laboratory data, it was found that none of the 82 patients who survived hospitalisation and were available for examination had GCA or PMR. Moreover, review of the retrospective data available from hospital records of the total consecutive 315 patients with AA/D failed to find any patient with a diagnosis of GCA/PMR. In conclusion, the present study did not find an increased prevalence of GCA/PMR among a cohort of Israeli patients with AA/D. Therefore, it is suggested that a thorough investigation aiming to diagnose GCA/ PMR is not cost effective in most of the elderly patients presenting with AA/D. (Postgrad Med F 2000;76:409-411)
\end{abstract}

Chaim Sheba Medical Center, Tel-Hashomer, Sackler Faculty of Medicine, Tel Aviv University, Israel: Department of Medicine C M Ehrenfeld

R Bitzur

Y Sidi

H Gur

Department of Vascular Surgery J Schneiderman

Department of Cardiac Surgery A Smolinsky

Correspondence to: Dr Hanan Gur, Department of Medicine C, Chaim Sheba Medical Center, Tel

Hashomer, Israel 52621

(e-mail:

hanang@post.tau.ac.il)

Submitted 15 July 1999 Accepted 7 December 1999
Keywords: giant cell arteritis; polymyalgia rheumatica; aortic aneurysm; aortic dissection

Giant cell arteritis (GCA) is a vasculitis affecting medium sized arteries in patients older than 50 years of age and is closely identified with the temporal arteritis/polymyalgia rheumatica (PMR) syndrome of the elderly. Despite the systemic character of the disease, it may display a distinct preference for certain arterial territories with focal arteritic lesions emerging in the aorta and its major branches, leading to aortic arch syndrome with subclavian, axillary, and brachial artery involvement. ${ }^{2}$ Thus, Lie reviewed 72 cases of aortic and large vessel GCA, ${ }^{1}$ claiming that up to $15 \%$ of patients with temporal arteritis might have angiographic evidence of aortic or extracranial GCA. Although aortic aneurysms are usually a late complication of GCA, they can occur at any time during the course of the disease. ${ }^{3}$ Moreover, aortic insufficiency, ruptured aortic aneurysm, and aortic dissection might be the initial manifestation of a biopsy proved systemic GCA. ${ }^{3}$
Giant cell aortitis, first described in 1937, might involve the entire aorta, but complications are usually related to thoracic involvement. ${ }^{3}$ Patients with giant cell aortitis may be asymptomatic, or present with aortic arch syndrome, dilatation of the aorta, aortic aneurysm, aortic dissection, sudden rupture, or aortic insufficiency. ${ }^{4}$ Thoracic aneurysms are usually fusiform and can be complicated by dissection in up to $50 \%$ of patients. This serious outcome of GCA has been recently reviewed by Liu et al who described 24 cases of aortic dissection in patients with GCA, $46 \%$ of whom presented with a catastrophic aortic dissection and no prior history of GCA. Dissection tears occurred primarily in the proximal aorta in $85 \%$ of the cases and hypertension was concomitant in $77 \%$ of patients. ${ }^{5}$ In another recent study, 16/41 patients with GCA involving the thoracic aorta developed acute aortic dissection. ${ }^{6}$ Other complications in this high risk group included aortic insufficiency, heart failure, and death. It is suggested that corticosteroid therapy might prevent fatal outcome with aortic rupture or dissection, but this notion has not been addressed by prospective studies. $^{4}$

Based on these observations and a recent population based study, it has recently been claimed that GCA is associated with a markedly increased risk of aortic aneurysm formation or rupture. ${ }^{37}$ Thus, in a population based cohort study from Minnesota, 11/96 patients $(11.4 \%)$ developed thoracic aortic aneurysm and the incidence of thoracic aortic aneurysm, aortic dissection, or both (AA/D) was reported to be 17.3 times higher in patients with GCA compared with the general population. ${ }^{7}$ In two patients the diagnosis of aortic aneurysm was concomitant to the diagnosis of GCA, while in the remaining nine patients the time after the diagnosis of GCA was 5.8 years. Six of these patients died suddenly of aortic dissection. In five other patients an abdominal aortic aneurysm was diagnosed, with a median of 2.5 years after the diagnosis of GCA. Similarly, the incidence of abdominal aortic aneurysm was 2.4 times higher than those of the general population.

The increased mortality associated with aortic aneurysmal disease associated with GCA has led to an increase in the concern about the significance of this association. ${ }^{3}$ A relevant question is how prevalent are GCA associated $\mathrm{AA} / \mathrm{D}$ among the general elderly population. Should we screen any patient with AA/D for the presence of GCA? 
The aim of the present study, therefore, was to examine the incidence of GCA in a cohort of patients with AA/D.

\section{Patients and methods}

The records of all patients admitted to the Chaim Sheba Medical Center with the diagnosis of $\mathrm{AA} / \mathrm{D}$ during a four year period were reviewed. In addition, follow up information was obtained by inviting the patients to the outpatient clinic, where they were interviewed, examined, and their erythrocyte sedimentation rate (Westegren) was measured. GCA and the related PMR were diagnosed using the American College of Rheumatology classification criteria for the diagnosis of $\mathrm{GCA}^{8}$ and the Healey criteria for the diagnosis of PMR, ${ }^{9}$ respectively. Patients with traumatic dissection or those with AA/D associated with congenital diseases (for example, Marfan's syndrome) or syphilis were excluded from the analysis.

\section{Results}

\section{PATIENT CHARACTERISTICS}

During the four year study period, 315 patients with the diagnosis of $\mathrm{AA} / \mathrm{D}$ were identified. Abdominal AA/D was the most common diagnosis, occurring in 199 patients (63\%), compared with thoracic AA/D (90 patients, $29 \%$ ). In 26 patients $(8 \%)$ the location was not specified. Sixteen patients ( $5 \%$ ) with either congenital $(n=11)$ or traumatic $(n=5)$ AA/D were excluded. Seven patients $(2 \%)$ were overseas tourists, and were lost to a further follow up. According to the official registry, the total mortality of the cohort until the date of examination was 35\% (110 patients).

The remaining 182 patients were invited for examination by mail, but information on the prevalence of GCA/PMR as obtained by clinical examination and interview was available in only $82 / 182$ patients ( $45 \%)$. Of the 82 patients examined, 39 (47\%) had thoracic AA/D and 43 (53\% ) had abdominal AA/D.

The mean age at the time of the examination was 71.5 (4) years, without a difference between patients with thoracic or abdominal AA/D (69 (4) and 70 (4) years, respectively). The majority of the patients $(70.5 \%)$ were males, with a higher frequency of males in patients with abdominal compared with thoracic $\mathrm{AA} / \mathrm{D}$ ( $83 \%$ v 58\%, respectively).

Table 1 Signs and symptoms suggesting GCA/PMR in 82 examined patients with $A A / D$

\begin{tabular}{lll}
\hline Symptom/sign & $\begin{array}{l}\text { Abdominal } A A / D \\
(n=43)\end{array}$ & $\begin{array}{l}\text { Thoracic } A A / D \\
(n=39)\end{array}$ \\
\hline $\begin{array}{l}\text { Previous diagnosis of GCA } \\
\text { Previous diagnosis of PMR }\end{array}$ & $\begin{array}{l}\text { None } \\
\text { Unexplained headache }\end{array}$ & $\begin{array}{l}\text { None } \\
\text { None }\end{array}$ \\
Temporal artery findings on examination & $4(9 \%)$ & $4(10 \%)$ \\
Erythrocyte sedimentation rate $>40 \mathrm{~mm} /$ hour & None & None \\
Girdle pain $>1$ month & $2(5 \%)$ & $2(5 \%)$ \\
Jaw claudication & $5(12 \%)$ & $3(8 \%)$ \\
Loss of vision & None & None \\
Unexplained fatigue & None & $1(2 \%)$ \\
Arthralgia & None & $1(2 \%)$ \\
Past or present corticosteroid therapy & $1(2 \%)$ & $1(2 \%)$ \\
Clinical diagnosis or American College of & None & None \\
Rheumatology classification criteria for GCA & None & None \\
Clinical diagnosis or Healey criteria for PMR & None & None \\
\hline
\end{tabular}

PREVALENCE OF GCA AND PMR IN PATIENTS WITH AORTIC ANEURYSM AND DISSECTION

After careful examination and assessment of clinical and laboratory data, it was found that none of the 82 patients who survived hospitalisation and were available for examination had GCA nor PMR. Moreover, review of the retrospective data available from hospital records of the total consecutive 315 patients with $\mathrm{AA} / \mathrm{D}$ failed to find any patient with a diagnosis of GCA/PMR. As can be seen in table 1, a few patients had symptoms and signs that were suggestive of GCA/PMR, but nevertheless could not meet clinical diagnosis nor classification criteria for diagnosis of GCA/PMR. ${ }^{89}$

\section{Discussion}

The present study aimed to examine the prevalence of GCA/PMR in a cohort of 315 consecutive patients with $\mathrm{AA} / \mathrm{D}$ admitted to the largest medical centre in Israel during a four year period. Both a retrospective analysis of all the in-hospital medical records and a prospective assessment of 82 patients who comprise about $45 \%$ of surviving patients failed to find even one patient fulfilling the classification criteria or clinical diagnosis for GCA/PMR. ${ }^{8} 9$ Thus, it is suggested by this report that in the population of $\mathrm{AA} / \mathrm{D}$ patients examined here, GCA/PMR did not play a significant part. Moreover, it is feasible to conclude that at least in the Israeli population, it is probably not cost effective to thoroughly investigate elderly patients presenting with AA/D, looking for an association with GCA/ PMR.

The main shortcoming of the present study is the lack of follow up data on the patients who had survived hospital, but failed to come to examination and assessment. However, since almost half of the available patients were assessed $(82 / 182)$ and none was found to have GCA/PMR, it is probably unlikely that the remaining patients had a significantly high prevalence of GCA/PMR. Nevertheless, this is an assumption that should be proved in further careful prospective studies.

The prevalence of GCA/PMR in patients with $\mathrm{AA} / \mathrm{D}$ has not been previously directly examined. Spence et al reported that the prevalence of GCA in patients with aortic aneurysm was $11.6 \% .^{10}$ However, they did include both GCA and Takayassu's arteritis. Rasmussen et $a l^{11}$ and Nitecki et $a l^{12}$ described a surgical pathological entity designated inflammatory aortic aneurysm which was found in $8 \%-10 \%$ of their patients with thoracic or abdominal aneurysms. It is currently not known whether this diagnosis correlates clinically to GCA/ PMR. Interestingly, in some patients with inflammatory aortic aneurysm the erythrocyte sedimentation rate was raised..$^{12}$ Moreover, the HLA-DRB1 locus was found to be a genetic component in both GCA, ${ }^{13}$ PMR, ${ }^{14}$ and inflammatory abdominal aortic aneurysms. ${ }^{15}$ Hence, it is possible that few patients of the current cohort had inflammatory aortic aneurysm, as $4 / 82(5 \%)$ of the patients had an unexplained raised erythrocyte sedimentation rate (table 1). However, it is a non-specific test, 
and cannot suggest without pathological data that these patients had inflammatory aortic aneurysm.

The main assumption of the present study, that GCA/PMR would be found relatively frequently in a cohort of patients with $\mathrm{AA} / \mathrm{D}$, was based on the recent reports on the significant association of GCA and AA/D. ${ }^{1357}$ The failure to find cases of GCA/PMR in the present report of $\mathrm{AA} / \mathrm{D}$ patients could reflect a lower incidence of GCA in the Israeli population, compared with Olmsted County, Minnesota. ${ }^{7}$ Indeed, the annual incidence rate for GCA in Israel has been estimated to be only $0.16-0.86 / 100000,{ }^{16}$ compared with $17 /$ 100000 in Olmsted County, ${ }^{17}$ although a more recent report on the prevalence of the GCA in Jerusalem metropolitan area showed an incidence of $10.2 / 100000 .^{18}$ Hence, it is suggested that different ethnic or genetic backgrounds and/or geographical/environmental factors are involved in the lower frequency of GCA in Israeli patients, and might affect different GCA subsets and patterns. Thus, it is possible that large vessel $\mathrm{GCA}^{12}$ is less frequent in Israeli patients. Indeed, none of the patients described in our recent report of GCA patients had large vessel GCA, nor AA/D. ${ }^{19}$

Although the present study has not revealed an association between AA/D and GCA in the Israeli population, it should be noted that in other populations such an association is possible and should be looked for.

In conclusion, our study did not find an increased prevalence of GCA/PMR among a cohort of Israeli patients with $\mathrm{AA} / \mathrm{D}$. Therefore, it is suggested that a thorough investigation aiming at diagnosing GCA/PMR is not cost effective in most of the elderly patients presenting with $\mathrm{AA} / \mathrm{D}$, at least in the Israeli population.
1 Lie JT. Aortic and extracranial large vessel giant cell arteritis: a review of 72 cases with histopathologic documentation. Semin Arthritis Rheum 1995;24:422-31.

2 Weyand CM, Brack A, Martinez-Talboda V, et al. Disease pattern and large vessel giant cell arteritis. Arthritis Rheum 1998;41:S347.

3 Evans J, Hunder GG. The implication of recognizing largevessel involvement in elderly patients with giant cell arteritis. Curr Opin Rheumatol 1997;9:37-40.

4 Le Tourneau T, Millaire A, Asseman P, et al. Aortitis in Horton disease. Review of the literature. Ann Med Interne (Paris) 1996;147:361-8.

5 Liu G, Shupak R, Chiu BKY. Aortic dissection in giant cell arteritis. Semin Arthritis Rheum 1995;25:160-71.

6 Evans JM, Bowles CA, Bjornson J, et al. Thoracic aortic aneurysm and rupture in giant cell arteritis. A descriptive study of 41 cases. Arthritis Rheum 1994;37:1539-47.

7 Evans JM, O'Fallon WM, Hunder CG. Increased incidence of aortic aneurysm and dissection in giant cell (temporal) arteritis. Ann Intern Med 1995;122:502-7.

8 Lie JT. American College of Rheumatology Subcommittee on classification of vasculitis. Arthritis Rheum 1990;33: 1074-87.

9 Healey LA. Long-term follow-up of polymyalgia rheumatica - evidence for synovitis. Semin Arthritis Rheum 1984;13:322-8.

10 Spence RK, Estella F, Gisser S, et al. Thoracic aortic aneurysm secondary to giant cell arteritis: a reappraisal of etiolrysm secondary to giant cell arteritis: a reappraisal of etiol-
ogy, treatment and possible prevention. $\mathcal{F}$ Cardiovasc Surg ogy, treatment a

11 Rasmussen TE, Hallett WR Jr. Inflammatory aortic aneurysms. A clinical review with new perspectives in pathogenesis. Ann Surg 1997;225:155-64.

12 Nitecki SS, Hallett JW Jr, Stanson AW, et al. Inflammatory abdominal aortic aneurysms: a case control study. $\mathcal{F}$ Vasc Surg 1996;23:860-8.

13 Weyand CM, Hicok KC, Hunder GG, et al. The HLA-DRB1 locus as a genetic component in giant cell arteritis. Mapping of a disease-linked sequence motif to the arteritis. Mapping of a disease-linked sequence motif to the
antigen binding site of the HLA-DR molecule. 7 Clin Invest antigen binding site

14 Weyand CM, Hunder NN, Hicok KC, et al. HLA-BRB1 alleles in polymyalgia rheumatica, giant cell arteritis, and rheumatoid arthritis. Arthritis Rheum 1994;37:514-20.

15 Rasmussen TE, Hallett JW Jr, Metzger RL, et al. Genetic risk factors in inflammatory abdominal aortic aneurysms: polymorphic residue 70 in the HLA-DRB1 gene as a key genetic element. $\mathcal{F}$ Vasc Surg 1997;25:356-64.

16 Friedman G, Friedman B, Benbassat J. Epidemiology of temporal arteritis in Israel. Isr F Med Sci 1982;18:241-4.

17 Machado EB, Michet CJ, Ballard DJ, et al. Trends in incidence and clinical presentation of temporal arteritis in Olmsted County, Minnesota, 1950-1985. Arthritis Rheum 1988;31:745-9.

18 Sonnenblick M, Nesher G, Friedlander Y, et al. Giant cell arteritis in Jerusalem: a 12 -year epidemiologic study. $\mathrm{Br} \mathcal{F}$ Rheumatol 1994;33:938-41.

19 Gur H, Rapman E, Ehrenfeld M, et al. Clinical manifestations of temporal arteritis: a report from Israel. $\mathcal{F}$ Rheumatol 1996;23:1927-31. 